\title{
Persistent infection of rhesus monkeys with 'Helicobacter macacae' and its isolation from an animal with intestinal adenocarcinoma
}

\begin{abstract}
Correspondence
James G. Fox

jgfox@mit.edu
\end{abstract}

Received 22 January 2010

Accepted 18 April 2010

\author{
Robert P. Marini, ${ }^{1}$ Sureshkumar Muthupalani, ${ }^{1}$ Zeli Shen, ${ }^{1}$ \\ Ellen M. Buckley, ${ }^{1}$ Cynthia Alvarado, ${ }^{1}$ Nancy S. Taylor, ${ }^{1}$ Floyd E. Dewhirst, ${ }^{2}$ \\ Mark T. Whary, ${ }^{1}$ Mary M. Patterson ${ }^{1}$ and James G. Fox ${ }^{1}$ \\ ${ }^{1}$ Division of Comparative Medicine, Massachusetts Institute of Technology, 77 Massachusetts \\ Avenue, Cambridge, MA 02139, USA \\ ${ }^{2}$ Department of Molecular Genetics, The Forsyth Institute, 140 The Fenway, Boston, MA 02115, USA
}

A novel helicobacter, 'Helicobacter macacae', was previously isolated from a colony of rhesus and cynomolgus monkeys in which diarrhoea from chronic idiopathic colitis was enzootic. A survey performed in a second colony of rhesus monkeys without a history of chronic diarrhoea determined that $57 \%$ were faecal-culture positive for Helicobacter species. Ten years after the survey, one of the animals from which ' $H$. macacae' had been isolated, a 23-year-old, intact male rhesus monkey (Macaca mulatta), presented with partial inappetence and progressive weight loss. Subsequent evaluation of the monkey revealed anaemia, hypoproteinaemia, hypoalbuminaemia and a palpable abdominal mass. Contrast radiography suggested partial intestinal obstruction. The animal was euthanized and a diagnosis was made of intestinal adenocarcinoma of the ileocaecocolic junction with metastasis to regional lymph nodes and liver. Microaerobic culture of caecal tissue yielded a helicobacter organism identified as ' $H$. macacae' by $16 \mathrm{~S}$ rRNA gene sequencing - the same species of bacteria isolated 10 years previously. The liver, small intestine and colon were also positive by PCR for Helicobacter species. Intestinal adenocarcinoma is the most common malignancy of aged macaques. Faeces or caecal tissue from five out of five monkeys that remained from the original cohort and that were colonized with ' $H$. macacae' in the initial survey were positive for the organism. The apparent persistence of ' $H$. macacae' in these animals, the isolation of the bacterium from animals with colitis and the recognition of the importance of inflammation in carcinogenesis raise the possibility of an aetiological role in the genesis of intestinal adenocarcinoma in aged rhesus monkeys.

\section{INTRODUCTION}

Inflammatory and neoplastic conditions across several species have been attributed to enterohepatic helicobacters. Helicobacter hepaticus is associated with chronic active hepatitis, hepatic adenoma and adenocarcinoma in susceptible mice (Fox et al., 1994, 1996). In a number of strains of immune-compromised mice and stocks and strains of defined flora and gnotobiotic mice, this organism can cause typhlocolitis, enterocolitis and lower bowel cancer (Rogers \& Fox, 2004). A novel Helicobacter species has been isolated from the inflamed colon of cotton-top tamarins, which are predisposed to developing inflammatory bowel disease (IBD) and colon cancer (Saunders et al., 1999). In macaques, Helicobacter cinaedi has been isolated from the colon, liver and mesenteric lymph node of a rhesus monkey with chronic colitis and hepatitis (Fox et al.,

Abbreviation: IBD, inflammatory bowel disease. 2001a). Both H. cinaedi and Helicobacter fennelliae have been used experimentally to induce diarrhoea and bacteraemia in infant pigtail macaques (Flores et al., 1990). In 2001, our laboratory isolated two novel Helicobacter species from colonic tissue of monkeys in a colony in which chronic idiopathic colitis and diarrhoea were enzootic (Fox et al., 2001b). The two organisms were designated Helicobacter sp. rhesus type 1 and 2, the former having been isolated from two animals with mild colitis but without diarrhoea and the latter from monkeys with diarrhoea and moderate to severe colitis. Slender, spiral, argyrophilic bacteria consistent with Helicobacter species were also observed in samples of colonic mucosa. Evaluation of faecal cultures with confirmation of Helicobacter species by a genus-specific PCR assay in a second colony of rhesus monkeys in which diarrhoea was not enzootic yielded Helicobacter species in 20/35 animals (57\%). Of these 20 enteric helicobacters, 13 were presumptively identified as 'Helicobacter macacae' by RFLP analysis; 
three of these strains were confirmed to be ' $H$. macacae' by $16 \mathrm{~S}$ rRNA gene sequencing (Fox et al., 2007). Isolates of rhesus type 1 from both colonies were used for full characterization of the organism; it was subsequently named 'Helicobacter macacae' (Fox et al., 2007). The organism has also been isolated from the ileum and liver of a baboon (García et al., 2006) and its DNA has been identified in a stool sample from a child with gastroenteritis (Haggerty et al., 2005).

A decade after the original survey, a rhesus monkey from the second colony from which ' $H$. macacae' had been isolated was diagnosed with intestinal adenocarcinoma. Culture of the caecum adjacent to the neoplasm yielded an organism identical to that isolated previously. In this study, we have described the potential relationship between this organism and intestinal cancer, and report the positive findings of ' $H$. macacae' colonization in a second survey for enteric helicobacters in rhesus monkeys remaining from the original cohort.

\section{METHODS}

Animals. A 23-year-old, intact male, singly housed rhesus monkey (Macaca mulatta, animal no. 93-11) showed clinical signs of progressive inappetence, decreased faecal production and weight loss over several weeks. Pertinent physical examination findings included poor body condition and moderate thoracolumbar kyphosis. The results of a complete blood count and serum chemistry analysis included mature neutrophilia, anaemia, hypoalbuminaemia and hypoproteinaemia. A radiographic contrast examination was consistent with partial intestinal obstruction. The animal was euthanized and a complete necropsy was performed. Tissues were fixed in $10 \%$ neutral-buffered formalin, processed routinely and stained with haematoxylin and eosin. The liver, ileocaecal junction, caecum and colon were also stained with Warthin-Starry stain for argyrophilic bacteria. Representative samples of various gastrointestinal tissues (stomach, jejunum, ileum, ileocaecal junction, caecum, colon and liver) were collected in freeze medium (1-2 $\mathrm{ml} 20 \%$ glycerol in Brucella broth) and stored at $-70{ }^{\circ} \mathrm{C}$ for subsequent culture.

Ten additional rhesus monkeys ( $M$. mulatta, eight males and two females) remained from the original cohort of 35 animals tested in 1999. They were singly housed in stainless steel cages $(31 \times 29 \times 64$ inches) in an Association for Assessment and Accreditation of Laboratory Animal Care International-accredited animal facility. Animals were fed ad libitum with commercial primate chow (Lab Diet 5038; PMI Nutrition International) and daily fruits, vegetables and treats. All animals were used in cognitive neuroscience research and, in accordance with Institutional Animal Care and Use Committee-approved protocols, had periodic but limited restriction of access to water. Animals that were off-study had water available ad libitum. The vivarium had 10-15 complete air changes $\mathrm{h}^{-1}$, a $12 \mathrm{~h}$ light: dark cycle, and temperature and humidity levels of $72-78{ }^{\circ} \mathrm{F}$ and $30-70 \%$, respectively.

Microbiology. Faecal samples collected from the cage pans of nine monkeys were placed onto the following media: Skirrow's medium, Campy CVA agar, blood agar and Glaxo. In a tenth animal (94-9) and in the animal with adenocarcinoma, evaluation for helicobacter was achieved using tissue samples from the ileum, colon and caecum obtained at necropsy. Microaerobic culture of faeces or tissue was performed as described previously (Fox et al., 2001b). Cultures were restreaked periodically onto fresh medium after the initial inoculation. Plates with optimum growth were used for DNA extraction; colonies were also Gram stained, evaluated for morphology and collected in freeze medium for storage at $-70{ }^{\circ} \mathrm{C}$. Biochemical analysis was performed as described previously (Fox et al., 2001b).

DNA extraction. The High Pure PCR Template Preparation kit (Roche Molecular Biochemicals) was used to extract DNA from bacterial pellets as described previously (Fox et al., 2007).

PCR amplification. DNA was amplified by Helicobacter genusspecific primers (C97 and CO5E) and a $1200 \mathrm{bp}$ product was separated using gel electrophoresis on a $1 \%$ agarose gel, as described previously (Fox et al., 2007).

RFLP. RFLP analysis of Helicobacter species DNA was performed using Alu $\mathrm{I}$ and HhaI digestion. The restriction patterns of the digested products were compared after separation in a VisiGel $6 \%$ separation matrix as described previously (Fox et al., 2007).

165 rRNA gene sequencing. Purified DNA from a PCR of the caecal isolate of the animal with adenocarcinoma was sequenced with an ABI Prism cycle sequencing kit (BigDye Terminator Cycle Sequencing kit with AmpliTaq DNA polymerase FS; Perkin Elmer) as described previously (Fox et al., 2007).

\section{RESULTS AND DISCUSSION}

\section{Necropsy and histopathology}

Post-mortem examination of animal 93-11 revealed the presence of moderate small intestinal and caecal distension proximal to a focal annular stricture at the ileocaecal junction (Fig. 1a, arrow). On dissection, the ileocaecal junction exhibited a focally extensive $(2-3 \mathrm{~cm}$ diameter), firm, raised, nodular thickening of the intestinal wall with intraluminal protrusion and cavitating constriction of the central core of the mass giving a 'napkin ring'-like appearance (Fig. 1b). The mass was associated with visible haemorrhage and mucosal ulceration. Other pertinent gross findings included prominent, multiple, variably sized (0.5-2.5 cm diameter), tan to yellow, slightly nodular to flat or depressed, discrete to vaguely demarcated foci involving all liver lobes both on the capsular surfaces and on cut sections of the parenchyma (Fig. 1c).

Histologically, the primary intestinal mass was diagnosed as tubular adenocarcinoma on the basis of the predominant morphological pattern involving the terminal ileum and extension into the ileocaecal valve and adjacent proximal caecum (Fig. 1d-f). The neoplastic tubulo-acinar glandular structures and occasional solid nests/cords or clusters of neoplastic cells were highly invasive with transmural involvement and effacement (Fig. 1e, f). The neoplasm was moderately well differentiated and cytologically exhibited a mixed cellular pattern ranging from glands/acini lined by tall, deeply basophilic columnar cells with a large prominent nucleus to tubulo-acinar structures and loose aggregate clusters composed of cells with a pale staining to clear, small, finely vacuolated cytoplasm and a small, hyperchromatic, eccentric nucleus (Fig. 1f, g). Within and along the margins of the mass, there was 
variable moderate chronic to active lymphoplasmacytic and neutrophilic inflammation, multifocal mucosal erosion/ulceration, necrosis, haemorrhage, amyloidosis and moderate reactive fibrosis (scirrhous response). In multiple examined sections, a variable intermingling of foveolar and glandular hyperplasia to low-grade dysplasia/adenomatous glands transitioning to invasive carcinoma was present within and along the margins of the mass. Warthin-Starry staining of the ileocaecal junction, caecum and colon from both neoplastic and non-neoplastic regions demonstrated mats of argyrophilic, small, pleomorphic (spiral to occasionally short, rod-like) bacteria closely adherent to the surface epithelium and occasionally within glandular/ crypt lumens (Fig. 1h). In some areas of the tumour and adjacent normal mucosa, moderate numbers of long, argyrophilic, spiral to filamentous bacteria $(8-15 \mu \mathrm{m}$ in length) mixed with a few small spiral bacteria were also observed (Fig. 1h, inset). In addition, the ileocaecal adenocarcinoma existed on a background of moderate, diffuse, lymphoplasmacytic ileitis, typhlitis and amyloidosis. There was prominent effacing metastasis of the neoplastic glands into the adjacent mesentery and local ileocaecal lymph nodes with similar cytological characteristics as those observed in the ileocaecal mass. The liver masses were histologically classified as metastatic intestinal adenocarcinoma and of note was the fact that the neoplastic foci in the liver were more solid in pattern with occasional tubulo-alveolar differentiation and visible intraluminal mucus (Fig. 1i). Warthin-Starry staining of the liver and ileocaecal lymph node did not reveal the presence of any argyrophilic spiral (short or long) bacteria in either neoplastic or non-neoplastic foci. Additional liver lesions included moderate, random, macrovesicular, hepatocellular fatty change, amyloidosis and mild chronic portal hepatitis. There was mild lymphocytic colitis and gastritis; moderate amyloidosis was seen in the colon, stomach, spleen, lymph nodes and adrenal gland. In a second animal (94-9, see Table 1) evaluated at necropsy, moderate ileitis and mild colitis were observed. ' $H$. macacae' was isolated from the caecum.

\section{Microbiology}

The results of microbiological samples of faeces or caecum from the 10 rhesus monkeys are shown in Table 1. The organisms were Gram-negative, slender and slightly curved. ' $H$. macacae' was isolated from the faeces of all four animals from which it had been isolated in 1999 and from tissue culture of the caecum of both animals evaluated at necropsy. 'H. macacae' was also isolated from the faeces of one animal that had been negative. Two distinct novel helicobacters were cultured from the faeces of the remaining four monkeys; two of these had been negative in 1999, one had a mixed culture of Campylobacter and Helicobacter, and the other had another novel Helicobacter that had not been characterized further. Both novel species were identical to organisms isolated previously from different animals by our laboratory (071672 - novel species 1, and 99-10781 - novel species 2).

\section{PCR}

All tissues obtained from the animal with adenocarcinoma were positive for Helicobacter species by PCR using genusspecific primers.

\section{RFLP}

The gel electrophoretogram of the AluI and HhaI digest of the $1200 \mathrm{bp}$ fragment PCR product from archival and current isolates of ' $H$. macacae' is shown in Fig. 2. Four different RFLP patterns were observed in the nine strains isolated from faeces and the two from caecal samples of rhesus monkeys in 2009. Two belong to ' $H$. macacae' patterns B and D. These patterns shared over $99 \%$ sequence identity in their $16 \mathrm{~S}$ rRNA gene; mutations at two AluI cutting sites contributed to the different RFLP patterns. The remaining two patterns were identical to those of isolates cultured previously from the colony and designated novel species 1 and 2.

\section{S rRNA gene sequencing}

The ' $H$. macacae' strain isolated from the caecum of the animal with adenocarcinoma had a $16 \mathrm{~S}$ rRNA gene sequence identical to that of the ' $H$. macacae' strain sequenced previously a decade earlier (Fig. 3). The relationship of this isolate with archival isolates is shown in Fig. 4.

This report has documented the persistent colonization of an enterohepatic helicobacter in rhesus monkeys and is the first isolation and demonstration of argyrophilic bacteria with helicobacter morphology in intimate association with intestinal adenocarcinoma in this species. It should be noted that, in a manner similar to existing information in the literature, we detected two major morphological forms of argyrophilic bacteria: one with a short spiral morphology and the other with a long, spiral to filamentous form in the ileocaecocolic junction of the animal diagnosed with intestinal carcinoma. These organisms have been classified historically as either spirochaetes (short spiral) or flagellates (long spiral). The identity of the so-called 'flagellates' is uncertain, as attempts to culture and isolate them have been largely unsuccessful. The short spiral bacteria in the intestine of macaques are now increasingly recognized as members of the species Helicobacter. Consequently, the role of these unidentified flagellates or long spiral forms in the causation of intestinal inflammation and progression to cancer is unknown (Zeller \& Takeuchi, 1982).

The identification of ' $H$. macacae' by PCR in the liver of our index case was significant. Its presence was thought to be a result of bacterial translocation from the gut by one of two mechanisms: bacterial transport in association with metastasis of neoplastic colon cancer cells (either 


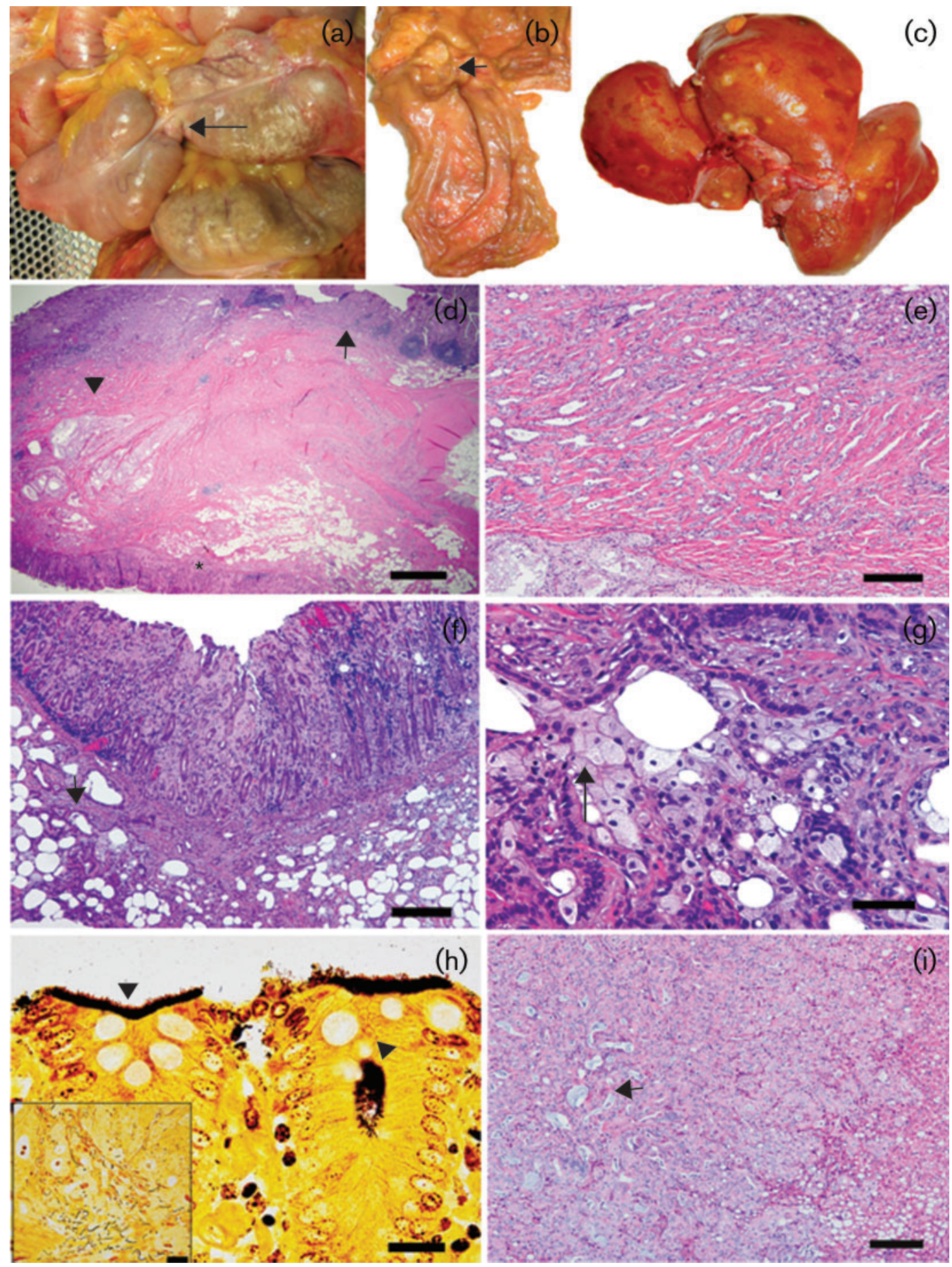

intracellularly or by superficial adherence) or independently of neoplastic cells either by the lymphatic route (through circulating lymph via lymph nodes) and/or by the haematogenous route (through the enterohepatic portal or systemic circulation) (Berg, 1995). Bacterial overgrowth and the associated loss of mucosal integrity in the context of inflammation are two key factors in bacterial translocation from the intestine to other tissues (Berg, 1995). As is common in colon cancers of humans and also observed in this report, intestinal cancers of rhesus monkeys are frequently associated with hepatic and lymph node metastasis. It is believed that this metastasis is aided by angiogenesis and lymphangiogenesis within the neoplasm followed by spread through circulating blood (systemic or portal) or lymph (via mesenteric nodes), respectively (Gout \& Huot, 2008). 
Fig. 1. Morphological findings in an animal (93-11) with chronic, persistent ' $H$. macacae' colonization. (a) In situ view of a focal intraluminar mass (arrow) causing an annular stricture at the ileocaecal junction with intestinal distension. (b) Gross dissection view of a focal 'napkin ring'-like mural thickening with mass effect (arrow) at the site of the ileocaecal stricture. (c) Multiple metastatic foci in the liver. (d) Low-magnification view of a haematoxylin and eosin-stained section at the region of the ileocaecal junction. The ileum (arrow), caecal valve (asterisk) and invasive glands (arrowhead) are indicated. Bar, $400 \mu \mathrm{m}$. (e) Highmagnification view of $(d)$ showing the variably sized, intramurally invasive, pale-staining, tubulo-acinar glandular structures with mild associated inflammation. Bar, $120 \mu \mathrm{m}$. (f) lleocaecal valve: mucosal thickening with hyperplastic and dysplastic/neoplastic glands and associated amyloidosis, mucosal erosion and haemorrhage. Note the multiple ectatic invasive glands (arrow) in the muscularis. Bar, $100 \mu \mathrm{m}$. (g) High-magnification view of (f) showing variable cellular differentiation features of the neoplastic cells. Note the invasive glands lined by a mixture of tall columnar eosinophilic cells with large nuclei to disorganized polygonal cells with finely vacuolated to clear cytoplasm and small eccentric nuclei (arrow). Bar, $40 \mu \mathrm{m}$. (h) Ileocaecal valve: mats of argyrophilic spiral to curved bacteria along the epithelial surface and within the glandular lumen (arrowheads). Bar, $12 \mu \mathrm{m}$. The inset shows a mixture of long argyrophilic, spiral to filamentous bacteria and small spirals. (i) Liver: representative metastatic focus in the liver with a predominant solid pattern and occasional tubulo-alveolar differentiation of metastatic glands and intraluminal mucus-like material (arrow). Bar, $100 \mu \mathrm{m}$.

While the long period between successive isolations of ' $H$. macacae' creates some uncertainty in the assertion of persistent colonization, the latter has ample precedence in the behaviour of enterohepatic helicobacters - that of subclinical and chronic infection persisting for the life of an immunocompetent host (Fox et al., 1996; Whary \& Fox, 2004). It is possible, however, that the isolation of these organisms does not reflect persistent colonization, but instead represents reinfection by organisms that the animal had cleared some time after the original isolation. Significantly, Helicobacter sp. rhesus type 1, the organism subsequently named ' $H$. macacae', was not associated with diarrhoea in macaques, but rather was found in animals with subclinical colitis. This is in contrast to Helicobacter sp. rhesus type 2, which was associated with chronic idiopathic colitis in young monkeys. The animals surviving from the original cohort had no history of enzootic diarrhoea and the persistence of their infection appeared to have had minimal clinical consequence for all but the animal with adenocarcinoma. This finding may parallel what is seen in humans infected with $H$. pylori, where comparatively few individuals with persistent infection actually develop clinical signs, and even fewer develop gastric cancer (Fox \& Wang, 2007).

While most animals included in this study were likely to have had persistent colonization from acquiring helicobacter infection as juveniles, three animals from which helicobacter was not isolated in the original survey became infected at some later time, one with ' $H$. macacae' and the others with novel helicobacters. Enterohepatic helicobacters have a faecal-oral route of transmission; because the animals in this report were singly housed, the most likely mode of infection for these latter three macaques was via fomites. Some animals may have been housed in cages that allowed finger-only contact with a neighbour; in these instances, contact transmission is possible. While our facility does not use pressure washing or hose cleaning of caging or holding areas, aerosolization and subsequent ingestion of organisms is possible in the facilities that utilize this sanitation regimen. The duration of infection of

Table 1. Helicobacters recovered from rhesus monkeys in 1999 and 2009

\begin{tabular}{|lll|}
\hline Animal ID & \multicolumn{1}{c|}{1999} & \multicolumn{1}{c|}{2009} \\
\hline $93-11^{*} \dagger$ & 'H. macacae', pattern B & 'H. macacae', pattern B \\
$94-9 \dagger$ & 'H. macacae', pattern B & 'H. macacae', pattern B \\
$98-5$ & 'H. macacae', pattern B & 'H. macacae', pattern B \\
$90-10$ & 'H. macacae', pattern B & 'H. macacae', pattern B \\
$94-10$ & Negative & 'H. macacae', pattern B \\
$98-6$ & 'H. macacae', pattern D & 'H. macacae', pattern D \\
$97-7$ & 'H. macacae', pattern D & 'H. macacae', pattern D \\
$95-9$ & Negative & Novel 1 \\
$98-7$ & Mixed Helicobacter/Campylobacter species & Novel 1 \\
$99-1$ & Novel Helicobacter species & Novel 1 \\
$92-8$ & Negative & Novel 2 \\
& & \\
\hline
\end{tabular}

${ }^{*}$ Monkey with adenocarcinoma.

$\nmid$ Monkeys from which ' $H$. macacae' was isolated using tissue. All other isolates are from faeces. 


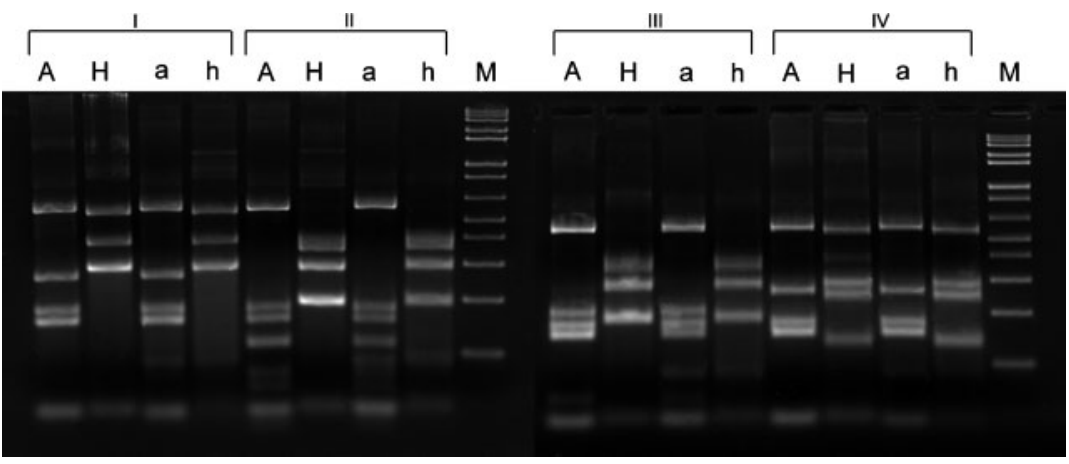

Fig. 2. Gel electrophoretogram of $A / u$ l and Hhal digests of the 16S rRNA gene $1200 \mathrm{bp}$ PCR fragment from current and archival rhesus monkey isolates. I, Helicobacter sp. monkey novel 1 ; II, ' $H$. macacae' pattern D; III, ' $H$. macacae' pattern B; IV, Helicobacter sp. monkey novel 2; A, 2009 Helicobacter isolates digested by Alul; a, 1999 Helicobacter isolates digested by Alul; H, 2009 Helicobacter isolates digested by Hhal; h, 1999 Helicobacter isolates digested by Hhal; $\mathrm{M}$, 100 bp ladder.

those animals newly identified as colonized with helicobacter is unknown.

The association of chronic enterocolitis with cancer is recognized in humans with IBD. Patients with both ulcerative colitis and Crohn's disease are at higher risk for the development of cancer (Rogers \& Fox, 2004). Among patients with ulcerative colitis, the incidence of adenocarcinoma is 15-20\% after 30 years of disease; for those with Crohn's disease, the 30 -year incidence is $8.3 \%$ (Boivin et al., 2003; Feagins et al., 2009). In mouse models of IBD, intestinal bacteria are required for inducing inflammation that culminates in neoplasia. In interleukin (IL)-10 and IL-2 deficient mice maintained under 'conventional' conditions, colitis progresses to adenocarcinoma. These lesions do not occur in mice in the germ-free state. In pathogen-free states in which only commensals have colonized the mice, the number and size of lesions diminish (Boivin et al., 2003; Rogers \& Fox, 2004).
Maggio-Price et al. (2009) demonstrated that infection with Helicobacter species was required to 'trigger' colitis and colorectal cancer in SmaD3 $3^{-1-} / \mathrm{Rag} 2$ mice. Using Rag2deficient mice, Erdman et al. (2009) showed that infection with $H$. hepaticus led to colitis, which progressed to cancer under the influence of tumour necrosis factor (TNF)- $\alpha$ and a Gr- $1^{+}$neutrophil-associated increase in inducible nitric oxide synthase expression. In these and similar studies, bacterial infection, its recognition by elements of the host immune system and subsequent downstream events in genetically susceptible mice led to chronic inflammation, hyperplasia, dysplasia and ultimately to adenocarcinoma.

Among the potential mechanisms by which inflammation may induce cancer are disruption of cellular homeostasis through cellular injury, alteration in cytoskeleton and cell proliferation kinetics, initiation of the innate and adaptive immune response, predisposition of tissue to genomic instability, and alteration of tumour suppressor and

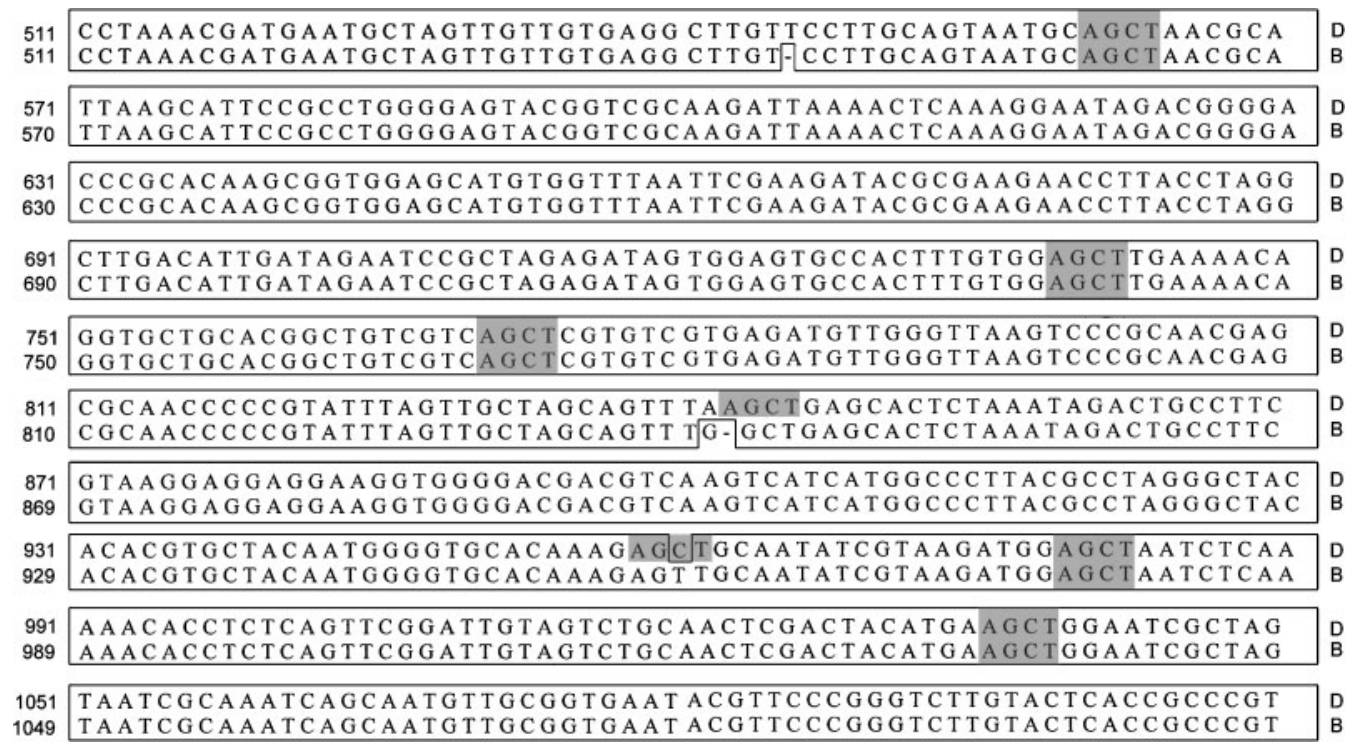

Fig. 3. Partial $16 \mathrm{~S}$ rRNA gene alignment of ' $H$. macacae' patterns $B$ and $D$. Identical sequences are boxed; shaded areas are cutting sites for Alul restriction enzyme. A deletion mutation at cutting site 843 and a $\mathrm{C} \rightarrow \mathrm{T}$ substitution mutation at site 960 contribute to the generation of these two patterns. 


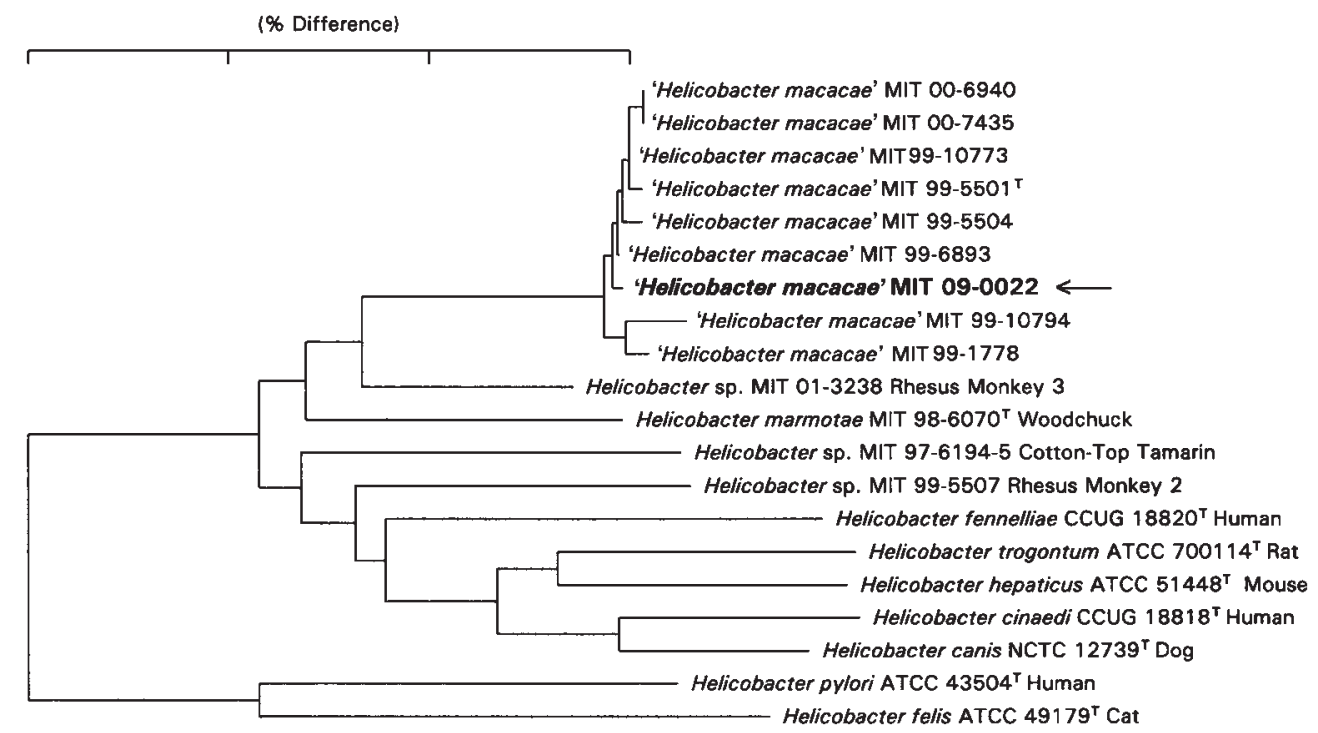

Fig. 4. Dendrogram demonstrating the similarity of sequences generated from the isolate collected from the animal with adenocarcinoma (MIT 09-0022, shown in bold) and other archival isolates. The bar shows $3 \%$ sequence differences as measured by the sum of the horizontal distances connecting taxa.

oncogene expression (Feagins et al., 2009; Hussain \& Harris, 2007). Mediators of these mechanisms include transcription factors, free radicals, cytokines, growth factors, prostaglandins and matrix metalloproteinases (Hussain \& Harris, 2007). Among the enterohepatic helicobacters, $H$. hepaticus is the organism that has most definitively been shown to induce a progression of inflammation to neoplasia, either through chronic active hepatitis to hepatocellular carcinoma (Fox et al., 1996; Ge et al., 2008; Ihrig et al., 1999; Rogers \& Fox, 2004) or IBD to colonic cancer (Erdman et al., 2003; Poutahidis et al., 2007 ) in genetically susceptible mice. In rodent models of colitis and cancer, host immune modulation by enteric bacteria, with IL-10, TNF- $\alpha$ and IL- 6 as pivotal cytokines, has been shown to influence neoplastic transformation of epithelium in microbially induced chronic inflammation (Erdman et al., 2003; Poutahidis et al., 2007). Using purified colonic epithelia from H. hepaticus-infected 129/ SvEv $\mathrm{Rag}^{-1-}$ mice, Poutahidis et al. (2007) also demonstrated dysregulation of the transforming growth factor (TGF)- $\beta$ signalling pathway, disruption of which is a feature of colorectal carcinoma in humans. The importance of TGF- $\beta$ has also been demonstrated in Helicobacter species-infected SmaD3 ${ }^{-1-}$ and SmaD3/Rag2 double-null mice (Maggio-Price et al., 2006, 2009).

A more thorough molecular characterization of known virulence determinants in ' $H$. macacae' and a better understanding of its effect on the host immune response, as well as confirmatory data from in situ hybridization studies, will help determine a causative rather than associative role for ' $H$. macacae' in intestinal adenocarcinoma. Studies of enteric flora in macaques in disease and health should also contribute to our understanding of
' $H$. macacae'-associated intestinal disease. A thorough evaluation of the macaque microbiome has recently been initiated by McKenna et al. (2008). In their study, DNA was isolated from stool, intestinal and colonic contents of rhesus monkeys, conserved regions of the 16S rRNA gene were amplified by PCR and the products were finally subjected to parallel pyrosequencing with subsequent operational taxonomic unit clustering and analysis. With this approach, the authors demonstrated diminished diversity of bacterial communities and a significant association of the bacterial family Campylobacteraceae with histopathological colitis. Helicobacter species were also detected in colonic mucosa. Recently, Gradel et al. (2009) demonstrated an increased hazard ratio for IBD in individuals who had experienced Campylobacter or Salmonella species-associated gastroenteritis. This risk, although highest in the first year after diagnosis, persisted throughout the entire follow-up period of up to 15 years. These findings support earlier studies demonstrating an increased odds ratio for IBD after bouts of infectious gastroenteritis in military personnel (Porter et al., 2008) and enhanced risk for Crohn's disease after Salmonella- and Campylobacter-associated enteritis (García Rodríguez et al., 2006). A study by Zhang et al. (2009) recently demonstrated an increased presence of Campylobacter concisus and anti-C. concisus antibodies in children with Crohn's disease. C. consisus was one of four non-Campylobacter jejuni campylobacters that contributed to a PCR positivity rate of $82 \%$ in isolates from Crohn's disease patients compared with $23 \%$ for controls. Taken together, these studies support the hypothesis that induction of enteritis with specific pathogens precipitates a cascade of events that may result in chronic inflammation and neoplasia in susceptible individuals. 
Intestinal adenocarcinoma is the most frequently diagnosed tumour of older macaques. In three separate surveys of the features of intestinal adenocarcinoma in macaques, all documented a high prevalence of intestinal tumours in aged animals $(>20$ years) and identified the ileocaecal junction as the most common site of neoplasia (Rodriguez et al., 2002; Uno et al., 1998; Valverde et al., 2000). Interestingly, this site is also a noted area of intestinal pathology in mouse models of helicobacter-induced IBD and lower bowel cancer (Cahill et al., 1997; Erdman et al., 2003, 2009; Maggio-Price et al., 2006). Common clinical features of weight loss, scant faecal production, anorexia, anaemia, hypoalbuminaemia and hypoproteinaemia were also described. Significantly, the animals in those reports had no known exposure to ionizing radiation or carcinogens. In one study, use of single-strand conformational polymorphism analysis to identify $K$-ras mutations, observed in approximately $40 \%$ of colonic adenocarcinomas of humans, failed to identify evidence of mutations in exon 1 or 2 in 10 macaques with intestinal adenocarcinoma (Valverde et al., 2000). In the current study, the persistence of ' $H$. macacae' in an animal with intestinal adenocarcinoma and accompanying colitis and the demonstration of argyrophilic organisms at the site of the lesion, as well as the many examples of helicobacter-associated tumorigenesis in other species, raise the intriguing possibility of a causative or contributory role for ' $H$. macacae'. The combined strategies of microbial community analysis in specific disease states, virulence factor characterization of specific micro-organisms, evaluation of tumour tissue for known cancer-associated genes and development of probes for in situ demonstration of infectious agents promise to provide important insights into enteric infection, inflammation and the role of bacteria in intestinal cancer.

\section{ACKNOWLEDGEMENTS}

This work was supported by grants RO1 CA 67529, T32 RR07036 and P30 - ES02109.

\section{REFERENCES}

Berg, R. D. (1995). Bacterial translocation from the gastrointestinal tract. Trends Microbiol 3, 149-154.

Boivin, G. P., Washington, K., Yang, K., Ward, J. M., Pretlow, T. P., Russell, R., Besselsen, D. G., Godfrey, V. L., Doetschman, T. \& other authors (2003). Pathology of mouse models of intestinal cancer: consensus report and recommendations. Gastroenterology 124, 762777.

Cahill, R. J., Foltz, C. J., Fox, J. G., Dangler, C. A., Powrie, F. \& Schauer, D. B. (1997). Inflammatory bowel disease: an immunitymediated condition triggered by bacterial infection with Helicobacter hepaticus. Infect Immun 65, 3126-3131.

Erdman, S. E., Poutahidis, T., Tomczak, M., Rogers, A. B., Cormier, K., Plank, B., Horwitz, B. H. \& Fox, J. G. (2003). CD ${ }^{+}$CD $25^{+}$regulatory $\mathrm{T}$ lymphocytes inhibit microbially induced colon cancer in Rag2deficient mice. Am J Pathol 162, 691-702.
Erdman, S. E., Rao, V. P., Poutahidis, T., Rogers, A. B., Taylor, C. L., Jackson, E. A., Ge, Z., Lee, C. W., Schauer, D. B. \& other authors (2009). Nitric oxide and TNF- $\alpha$ trigger colonic inflammation and carcinogenesis in Helicobacter hepaticus-infected, Rag2-deficient mice. Proc Natl Acad Sci U S A 106, 1027-1032.

Feagins, L. A., Souza, R. F. \& Spechler, S. J. (2009). Carcinogenesis in IBD: potential targets for the prevention of colorectal cancer. Nat Rev Gastroenterol Hepatol 6, 297-305.

Flores, B. M., Fennell, C. L., Kuller, L., Bronsdon, M. A., Morton, W. R. \& Stamm, W. E. (1990). Experimental infection of pig-tailed macaques (Macaca nemestrina) with Campylobacter cinaedi and Campylobacter fennelliae. Infect Immun 58, 3947-3953.

Fox, J. G. \& Wang, T. C. (2007). Inflammation, atrophy, and gastric cancer. J Clin Invest 117, 60-69.

Fox, J. G., Dewhirst, F. E., Tully, J. G., Paster, B. J., Yan, L., Taylor, N. S., Collins, M. J., Jr, Gorelick, P. L. \& Ward, J. M. (1994). Helicobacter hepaticus sp. nov., a microaerophilic bacterium isolated from livers and intestinal mucosal scrapings from mice. J Clin Microbiol 32, 1238-1245.

Fox, J. G., Li, X., Yan, L., Cahill, R. J., Hurley, R., Lewis, R. \& Murphy, J. C. (1996). Chronic proliferative hepatitis in A/JCr mice associated with persistent Helicobacter hepaticus infection: a model of helicobacter-induced carcinogenesis. Infect Immun 64, 1548-1558.

Fox, J. G., Handt, L., Sheppard, B. J., Xu, S., Dewhirst, F. E., Motzel, S. \& Klein, H. (2001a). Isolation of Helicobacter cinaedi from the colon, liver, and mesenteric lymph node of a rhesus monkey with chronic colitis and hepatitis. J Clin Microbiol 39, 1580-1585.

Fox, J. G., Handt, L., Xu, S., Shen, Z., Dewhirst, F. E., Paster, B. J., Dangler, C. A., Lodge, K., Motzel, S. \& Klein, H. (2001b). Novel Helicobacter species isolated from rhesus monkeys with chronic idiopathic colitis. J Med Microbiol 50, 421-429.

Fox, J. G., Boutin, S. R., Handt, L. K., Taylor, N. S., Xu, S., Rickman, B., Marini, R. P., Dewhirst, F. E., Paster, B. J. \& other authors (2007). Isolation and characterization of a novel helicobacter species, "Helicobacter macacae", from rhesus monkeys with and without chronic idiopathic colitis. J Clin Microbiol 45, 4061-4063.

Garcia, A., Xu, S., Dewhirst, F. E., Nambiar, P. R. \& Fox, J. G. (2006). Enterohepatic Helicobacter species isolated from the ileum, liver and colon of a baboon with pancreatic islet amyloidosis. J Med Microbiol 55, 1591-1595.

García Rodríguez, L. A., Ruigómez, A. \& Panés, J. (2006). Acute gastroenteritis is followed by an increased risk of inflammatory bowel disease. Gastroenterology 130, 1588-1594.

Ge, Z., Schauer, D. B. \& Fox, J. G. (2008). In vivo virulence properties of bacterial cytolethal-distending toxin. Cell Microbiol 10, 1599-1607.

Gout, S. \& Huot, J. (2008). Role of cancer microenvironment in metastasis: focus on colon cancer. Cancer Microenviron 1, 69-83.

Gradel, K. O., Nielsen, H. L., Schonheyder, H. C., Ejlertsen, T., Kristensen, B. \& Nielsen, H. (2009). Increased short- and long-term risk of inflammatory bowel disease after Salmonella or Campylobacter gastroenteritis. Gastroenterology 137, 495-501.

Haggerty, T. D., Perry, S., Sanchez, L., Perez-Perez, G. \& Parsonnet, J. (2005). Significance of transiently positive enzyme-linked immunosorbent assay results in detection of Helicobacter pylori in stool samples from children. J Clin Microbiol 43, 2220-2223.

Hussain, S. P. \& Harris, C. C. (2007). Inflammation and cancer: an ancient link with novel potentials. Int J Cancer 121, 2373-2380.

Ihrig, M., Schrenzel, M. D. \& Fox, J. G. (1999). Differential susceptibility to hepatic inflammation and proliferation in AXB recombinant inbred mice chronically infected with Helicobacter hepaticus. Am J Pathol 155, 571-582. 
Maggio-Price, L., Treuting, P., Zeng, W., Tsang, M., BielefeldtOhmann, H. \& Iritani, B. M. (2006). Helicobacter infection is required for inflammation and colon cancer in SMAD3-deficient mice. Cancer Res 66, 828-838.

Maggio-Price, L., Treuting, P., Bielefeldt-Ohmann, H., Seamons, A., Drivdahl, R., Zeng, W., Lai, L., Huycke, M., Phelps, S. \& other authors (2009). Bacterial infection of Smad3/Rag2 double-null mice with transforming growth factor- $\beta$ dysregulation as a model for studying inflammation-associated colon cancer. Am J Pathol 174, 317-329.

McKenna, P., Hoffmann, C., Minkah, N., Aye, P. P., Lackner, A., Liu, Z., Lozupone, C. A., Hamady, M., Knight, R. \& Bushman, F. D. (2008), The macaque gut microbiome in health, lentiviral infection, and chronic enterocolitis. PLoS Pathog 4, e20.

Porter, C. K., Tribble, D. R., Aliaga, P. A., Halvorson, H. A. \& Riddle, M. S. (2008). Infectious gastroenteritis and risk of developing inflammatory bowel disease. Gastroenterology 135, 781-786.

Poutahidis, T., Haigis, K. M., Rao, V. P., Nambiar, P. R., Taylor, C. L., Ge, Z., Watanabe, K., Davidson, A., Horwitz, B. H. \& other authors (2007). Rapid reversal of interleukin-6-dependent epithelial invasion in a mouse model of microbially induced colon carcinoma. Carcinogenesis 28, 2614-2623.

Rodriguez, N. A., Garcia, K. D., Fortman, J. D., Hewett, T. A., Bunte, R. M. \& Bennett, B. T. (2002). Clinical and histopathological evaluation of 13 cases of adenocarcinoma in aged rhesus macaques (Macaca mulatta). J Med Primatol 31, 74-83.

Rogers, A. B. \& Fox, J. G. (2004). Inflammation and cancer. I. Rodent models of infectious gastrointestinal and liver cancer. Am J Physiol Gastrointest Liver Physiol 286, G361-G366.

Saunders, K. E., Shen, Z., Dewhirst, F. E., Paster, B. J., Dangler, C. A. \& Fox, J. G. (1999). Novel intestinal Helicobacter species isolated from cotton-top tamarins (Saguinus oedipus) with chronic colitis. J Clin Microbiol 37, 146-151.

Uno, H., Alsum, P., Zimbric, M. L., Houser, W. D., Thomson, J. A. \& Kemnitz, J. W. (1998). Colon cancer in aged captive rhesus monkeys (Macaca mulatta). Am J Primatol 44, 19-27.

Valverde, C. R., Tarara, R. P., Griffey, S. M. \& Roberts, J. A. (2000). Spontaneous intestinal adenocarcinoma in geriatric macaques (Macaca sp.). Comp Med 50, 540-544.

Whary, M. T. \& Fox, J. G. (2004). Natural and experimental Helicobacter infections. Comp Med 54, 128-158.

Zeller, J. \& Takeuchi, A. (1982). Infection of the colon of the rhesus monkey by spiral-shaped organisms. Vet Pathol Suppl 7, 26-32.

Zhang, L., Man, S. M., Day, A. S., Leach, S. T., Lemberg, D. A., Dutt, S., Stormon, M., Otley, A., O'Loughlin, E. V. \& other authors (2009). Detection and isolation of Campylobacter species other than C. jejuni from children with Crohn's disease. J Clin Microbiol 47, 453-455. 